\title{
Periodic Behaviors in Multi-agent Systems with Input Saturation Constraints
}

\author{
Tao Yang, Ziyang Meng, Dimos V. Dimarogonas, Karl H. Johansson
}

\begin{abstract}
In this paper, we give conditions for the existence of periodic behaviors in a multi-agent system of identical discrete-time double integrators with input saturation constraints. If the feedback gain parameters of the controllers, which are based on relative state measurements of the agent itself and its neighboring agents, are bounded by a value depending on the largest eigenvalue of the Laplacian matrix, then the multi-agent system exhibits a periodic solution for certain initial conditions.
\end{abstract}

\section{INTRODUCTION}

The distributed coordination of a multi-agent system (MAS) has received substantial attention in recent years due to its wide application areas; we refer readers to pioneering works [1], [2]. One of problems in distributed coordination is consensus, where the goal is to design a distributed protocol based on the limited information of the agent itself and its neighboring agents, such that some variables of interest, either the state or partial state, become the same asymptotically. This problem has been extensively studied, e.g., some papers [3]-[16], or the recent books [17]-[19].

Most works in the literature consider the case where each agent does not have actuator saturation constraints. However, in almost every real world application, the actuator has bounds on its input, and thus actuator saturation constraint is important to study. The protocol design for achieving consensus for the case where each agent is subject to input saturation constraints is a challenging problem, and only few results are available, e.g., [20]-[23]. For the singleintegrator case, Li et al. [20] show that any linear protocol based on the relative state information, which solves the consensus problem for the case without input saturation constraints under fixed directed network topologies, also solves the global consensus problem in the presence of input saturation constraints. For neutrally stable dynamics and double-integrator cases, Meng et al. [21] propose a linear protocol to solve the global consensus problem for a MAS with input saturation constraints under fixed network topologies and time-varying network topologies. Yang et al. [22] solve the semi-global regulation of output synchronization for heterogeneous networks under fixed directed network topologies.

All the aforementioned references focus on continuoustime agent models. For the case where the agent models discrete-time, the authors [23] consider the global consensus problem in a multi-agent system of identical discrete-time

The authors are with ACCESS Linnaeus Centre, Royal Institute of Technology, Stockholm, Sweden. Email: \{taoyang, ziyangm, dimos, kallej\}@kth.se. This work has been supported in part by the Knut and Alice Wallenberg Foundation, the Swedish Research Council and EU HYCON2. double integrators with input saturation constraints under fixed undirected topologies. We show that if the feedback gain parameters of linear control laws, which solve the consensus problem for the case without input saturation constraints, satisfy a certain condition, then such linear control laws also solve the global consensus problem in the presence of input saturation constraints.

Dynamical behaviors including periodic solutions for general discrete-time dynamical systems have been extensively studied in [24]. In the earlier work [25], nontrivial periodic solutions were established for the closed-loop system of discrete-time double integrator with input saturation constraints under some locally stabilizing linear state feedback control laws. This earlier work [25] complements to the literature on periodic solutions for general discrete-time dynamical systems by considering the input saturation constraints. On the other hand, the input sequences for achieving periodic behaviors are such that the system is always in saturation, which implies that the system is equivalent to relay feedback systems. Thus, it also supplements the literature on continuous-time relay feedback systems [26]-[29], where fast switches and limit cycles (isolated periodic orbits) are investigated.

Motivated by the results in [23], [25], in this paper we investigate whether periodic solutions exist in the multi-agent system of identical discrete-time double integrators with input saturation constraints. More specifically, if the feedback gain parameters of control laws which solve the consensus problem without input saturation constraints satisfy a certain condition, then we show that there exist initial conditions that yield periodic solutions.

In the continuous-time setting, the existence of oscillatory behaviors in diffusively coupled systems has been considered in [30], while the synchronization of Kuramoto oscillators have been studied in [31], [32]. To the best of the authors' knowledge, periodic behaviors in a multiple discrete-time agent system have not been asserted before in the literature. The contribution of this paper is three-fold. First, it shows the importance of taking the input saturation constraints into account, which appear in almost every real world application. Second, it implies that the sufficient condition given in [23] for achieving global consensus has a necessary aspect, in the sense that the sufficient condition derived here for achieving periodic behavior is comparable to the condition in [23]. In addition, the derived periodic behaviors have potential application to networked systems of robots or autonomous vehicles. These include coverage control [33] and area surveillance [34], since the periodic patterns that are derived can be used in order to derive these type of 
behaviors in autonomous vehicle systems, whose inputs are generally bounded and thus saturated models thereof are both applicable and desirable.

The remainder of the paper is organized as follows. In Section II, some preliminaries and notations are introduced. In Section III, we motivate and formulate the problem. The main result is given in Section IV, where we give conditions for the existence of periodic solutions in the multi-agent system of identical discrete-time double integrators. The simulation example is presented in Section $\mathrm{V}$ to illustrate the result. Section VI concludes the paper.

\section{Preliminaries And Notations}

In this paper, we assume that the communication topology among the agents is described by a fixed undirected weighted graph $\mathscr{G}=(\mathscr{V}, \mathscr{E}, \mathscr{A})$, with the set of agents $\mathscr{V}=\{1, \ldots, N\}$, the set of undirected edges $\mathscr{E} \subseteq \mathscr{V} \times \mathscr{V}$, and a weighted adjacency matrix $\mathscr{A}=\left[a_{i j}\right] \in \mathbb{R}^{N \times N}$, where $a_{i j}=a_{i j}>0$ if and only if $(i, j) \in \mathscr{E}$ and $a_{i j}=0$ otherwise. We also assume that there are no self-loops, i.e, $a_{i i}=0$ for $i \in \mathscr{V}$. The set of neighboring agents of agent $i$ is defined as $\mathscr{N}_{i}=\{j \in$ $\left.\mathscr{V} \mid a_{i j}>0\right\}$. A path from node $i_{1}$ to $i_{k}$ is a sequence of nodes $\left\{i_{1}, \ldots, i_{k}\right\}$ such that $\left(i_{j}, i_{j+1}\right) \in \mathscr{E}$ for $j=1, \ldots, k-1$. An undirected graph is said to be connected if there exists a path between any pair of distinct nodes. A node is called a root if there exists a path to every other node. For a connected graph, every node is a root.

For an undirected weighted graph $\mathscr{G}$, a matrix $L=\{\ell\}_{i j} \in$ $\mathbb{R}^{N \times N}$ with $\ell_{i i}=\sum_{j=1}^{N} a_{i j}$ and $\ell_{i j}=-a_{i j}$ for $j \neq i$, is called Laplacian matrix associated with the graph $\mathscr{G}$. It is well known that the Laplacian matrix has the property that all the row sums are zero. If the undirected weighted graph $\mathscr{G}$ is connected, then $L$ has a simple eigenvalue at zero with corresponding right eigenvector $\mathbf{1}$ and all other eigenvalues are strictly positive. For such a case, all the eigenvalues of the Laplacian matrix can be ordered as $0=\lambda_{1}<\lambda_{2} \leq \ldots \leq \lambda_{N}$. The set of positive integers is denoted by $\mathbb{Z}^{+}$while the set of non-negative integer is denoted by $\mathbb{Z}$.

Let us now review the general discrete-time dynamical system, which can be found in [24, Chapter1]. Consider a discrete-time dynamical system

$$
x(k+1)=F(x(k)),
$$

where

- $x(k)=\left[x_{1}(k), x_{2}(k), \ldots, x_{q}(k)\right]^{\mathrm{T}}$ is the state of $q$ variables of the system at time instant $k$;

- $F(x)=\left[F_{1}(x), F_{2}(x), \ldots, F_{q}(x)\right]^{\mathrm{T}}$ are the $q$ functions governing the process.

Given an initial state $x(0)$, the sequences of states $\{x(0), x(1), \ldots\}$ is denoted by $O(x(0))$, and it is called the orbit or trajectory of the system starting from $x(0)$.

Definition 1 An orbit $O(x(0))$ is said to be periodic with a period $T \in \mathbb{Z}^{+}$if

$$
x(k+T)=x(k), \quad \forall k \in \mathbb{Z} .
$$

\section{Problem Formulation}

Consider a multi-agent system (MAS) of $N$ identical discrete-time double integrators described by

$$
\left[\begin{array}{l}
x_{i}(k+1) \\
v_{i}(k+1)
\end{array}\right]=\left[\begin{array}{ll}
1 & 1 \\
0 & 1
\end{array}\right]\left[\begin{array}{l}
x_{i}(k) \\
v_{i}(k)
\end{array}\right]+\left[\begin{array}{l}
0 \\
1
\end{array}\right] \sigma\left(u_{i}(k)\right), \quad i \in \mathscr{V},
$$

where $\sigma(u)$ is the standard saturation function: $\sigma(u)=$ $\operatorname{sgn}(u) \min \{1,|u|\}$.

\section{Assumption 1 The undirected graph $\mathscr{G}$ is connected.}

Consider state feedback control laws based on the agent state relative to that of neighboring agents with feedback gain parameters $\alpha$ and $\beta$ of the form

$u_{i}(k)=-\alpha \sum_{j \in \mathscr{N}_{i}} a_{i j}\left(x_{i}(k)-x_{j}(k)\right)-\beta \sum_{j \in \mathscr{N}_{i}} a_{i j}\left(v_{i}(k)-v_{j}(k)\right)$.

We then recall the following result from [35], for the MAS (3) without the input saturation constraints.

Lemma 1 [35] Assume that Assumption 1 is satisfied. Consider the MAS (3) in the absence of actuator saturation. Then the control law (4) solves the consensus problem if and only if

$$
0<\alpha<\beta<\frac{\alpha}{2}+\frac{2}{\lambda_{N}} .
$$

The above lemma gives a necessary and sufficient condition on feedback gain parameters for achieving consensus without input saturation constraints. In our previous paper [23], we showed that a subset of the control laws (4), which solve the consensus problem for the MAS (3) without input saturation constraints, also solve the global consensus problem in the MAS (3) with input saturation constraints, which is recapped in the following lemma.

Lemma 2 [23] Assume that Assumption 1 is satisfied. Consider the MAS (3) in the presence of actuator saturation. Then the control law (4) with

$$
0<\sqrt{3} \alpha<\beta<\frac{3}{2 \lambda_{N}}
$$

solves the global consensus problem.

However, the behavior of the multi-agent system of (3) under linear control laws (4) whose feedback gain parameters satisfy the condition (5) but do not satisfy the condition given by (6) is unknown. In this paper, we show that for some $\alpha$ and $\beta$ which satisfy (5), the multi-agent system of (3) under linear control laws (4) exhibits periodic solutions, which is formally defined as follows based on Definition 1 .

Definition 2 The multi-agent system (3) under linear control laws (4) exhibits a periodic solution with a period $T>0$, if for some initial conditions $x_{i}(0)$ and $v_{i}(0)$ for $i \in \mathscr{V}$, we have $x_{i}(T)=x_{i}(0)$ and $v_{i}(T)=v_{i}(0)$ for all $i \in \mathscr{V}$. 


\section{PERIODIC BEHAVIOR}

In this section, we shall show that if the feedback gain parameters $\alpha$ and $\beta$ of control laws (4) satisfy a certain condition in addition to (5), then the multi-agent system (3) under these control laws exhibits nontrivial periodic behaviors. The following theorem shows this.

Theorem 1 Assume that Assumption 1 is satisfied. Consider the MAS (3) and control laws (4). If $\alpha$ and $\beta$ satisfy

$$
0<\alpha<\beta<\min \left\{\frac{\alpha}{2}+\frac{2}{\lambda_{N}}, \frac{3}{2} \alpha\right\},
$$

then the multi-agent system (3) under control laws (4) exhibits a periodic solution with some period $T>0$.

Proof: Since the graph is connected, every agent is a root agent. Without loss of generality, we assume that the agent 1 is the root agent.

We shall prove the theorem by explicitly constructing periodic solutions with an even period $T=2 m$, where $m$ is some positive integer, which will be specified later in the proof.

Let us first define the following sets based on whether distance between agent $i$ and the root agent 1 is even or odd:

$$
S_{e}=\{i \mid d(i, 1)=2 d\}, \text { and } S_{o}=\{i \mid d(i, 1)=2 d+1\},
$$

where $d \in\{0,1, \ldots\}$.

The periodic solution that we will construct is such that all agents are always in saturation. and Moreover, the saturated input sequences are composed of 1 for the first $m$ steps, followed by -1 for the next $m$ steps for agent $i \in \mathscr{V}$, and the saturated input sequences are composed of -1 for the first $m$ steps, followed by 1 for the next $m$ steps for agent $i \in S_{o}$. That is,

$$
\begin{aligned}
& \left\{\begin{array}{c}
u_{i}(k) \geq 1, k=0, \ldots, m-1, \\
u_{i}(k) \leq-1, k=m, \ldots, 2 m-1, \quad i \in S_{e},
\end{array}\right. \\
& \left\{\begin{array}{c}
u_{i}(k) \leq-1, k=0, \ldots, m-1, \\
u_{i}(k) \geq 1, k=m, \ldots, 2 m-1, \quad i \in S_{o} .
\end{array}\right.
\end{aligned}
$$

In what follows, we shall show that the above $2 \mathrm{Nm}$ inequalities are satisfied for certain positive integer $m$ and initial conditions $x_{i}(0)$ and $v_{i}(0)$ for $i \in \mathscr{V}$ in three steps.

Step 1: For such a solution, we always have $v_{i}(T)=v_{i}(0)$ for all $i \in \mathscr{V}$. From the characteristic of the input sequences, it is also easy to obtain that

$$
x_{i}(2 m)=x_{i}(0)+2 m v_{i}(0)+m^{2}, \quad i \in S_{e},
$$

and

$$
x_{i}(2 m)=x_{i}(0)+2 m v_{i}(0)-m^{2}, \quad i \in S_{o} .
$$

In order to have $x_{i}(T)=x_{i}(0)$ for all $i \in \mathscr{V}$, we must have that $v_{i}(0)=-\frac{m}{2}$ for $i \in S_{e}$ and $v_{i}(0)=\frac{m}{2}$ for $i \in S_{o}$.

Step 2: In this step, we show that the $2 m$ inequalities (8) and (9) for each agent $i$, where $i \in \mathscr{V}$, can be reduced to only two inequalities by properly choosing some of initial conditions $x_{i}(0)$, where $i \in \mathscr{V}$.
For $k=1, \ldots, m$, due to the required characteristic of the saturated input sequences, we have

$$
\left\{\begin{array}{l}
x_{i}(k)=x_{i}(0)+k v_{i}(0)+\frac{k(k-1)}{2} \\
v_{i}(k)=v_{i}(0)+k, \quad i \in S_{e}
\end{array}\right.
$$

and

$$
\left\{\begin{array}{l}
x_{i}(k)=x_{i}(0)+k v_{i}(0)-\frac{k(k-1)}{2}, \\
v_{i}(k)=v_{i}(0)-k, \quad i \in S_{o} .
\end{array}\right.
$$

Step 2.1: Let us now consider the input for agent $j \in S_{o}$,

$$
\begin{aligned}
& u_{j}(k) \\
= & \alpha \sum_{i \in \mathscr{N}_{j}} a_{i j}\left(x_{i}(k)-x_{j}(k)\right)+\beta \sum_{i \in \mathscr{N}_{j}} a_{i j}\left(v_{i}(k)-v_{j}(k)\right) \\
= & \alpha \sum_{i \in \mathscr{N}_{j} \cap S_{e}} a_{i j}\left(x_{i}(k)-x_{j}(k)\right)+\beta \sum_{i \in \mathscr{N}_{j} \cap S_{e}} a_{i j}\left(v_{i}(k)-v_{j}(k)\right) \\
& +\alpha \sum_{i \in \mathscr{N}_{j} \cap S_{o}} a_{i j}\left(x_{i}(k)-x_{j}(k)\right)+\beta \sum_{i \in \mathscr{N}_{j} \cap S_{o}} a_{i j}\left(v_{i}(k)-v_{j}(k)\right) .
\end{aligned}
$$

Let us choose $x_{i}(0)=x_{j}(0)$ if $(i, j) \in \mathscr{E}$, where $i \in S_{o}$ and $j \in S_{o}$. This together with the fact that $v_{i}(0)=\frac{m}{2}$ for $i \in S_{o}$ imply that (10) is equivalent to

$$
=\alpha \sum_{i \in \mathscr{N}_{j} \cap S_{e}}^{u_{j}(k)} a_{i j}\left(x_{i}(k)-x_{j}(k)\right)+\beta \sum_{i \in \mathscr{N}_{j} \cap S_{e}} a_{i j}\left(v_{i}(k)-v_{j}(k)\right) .
$$

Similarly, due to the symmetric property, we can show that for agent $i \in S_{e}$, if we choose $x_{i}(0)=x_{j}(0)$ for $(i, j) \in \mathscr{E}$, where $i \in S_{e}$ and $j \in S_{e}$, we have

$$
=\alpha \sum_{j \in \mathscr{N}_{i} \cap S_{o}}^{u_{i}(k)} a_{i j}\left(x_{j}(k)-x_{i}(k)\right)+\beta \sum_{j \in \mathscr{N}_{i} \cap S_{o}} a_{i j}\left(v_{j}(k)-v_{i}(k)\right) .
$$

Step 2.2: Let us now focus on a particular edge $(i, j) \in \mathscr{E}$, where $i \in S_{e}$ and $j \in S_{o}$. We first note that $0<\alpha<\beta$ from (5). This implies:

$$
\beta>\alpha-\frac{1}{2} k \alpha
$$

for $k=0, \ldots, m-1$. This yields

$$
-\frac{\alpha m}{2}+\beta>\frac{1}{2} \alpha(-m-k+2) .
$$

Since $m-k-1 \geq 0$, multiplying the above inequality on both sides with $m-k-1$ yields:

$$
-\frac{\alpha m}{2}(m-k-1)+\beta(m-k-1) \geq \alpha\left[\frac{k(k-1)}{2}-\frac{(m-1)(m-2)}{2}\right] .
$$

This is equivalent to:

$$
\begin{aligned}
& a_{i j}\left\{\alpha\left[x_{i}(m-1)-x_{j}(m-1)\right]+\beta\left[v_{i}(m-1)-v_{j}(m-1)\right]\right\} \\
& \geq a_{i j}\left\{\alpha\left[x_{i}(k)-x_{j}(k)\right]+\beta\left[v_{i}(k)-v_{j}(k)\right]\right\}
\end{aligned}
$$

for $k=0, \ldots, m-1$ since $v_{i}(0)=-\frac{m}{2}$ for $i \in S_{e}, v_{j}(0)=\frac{m}{2}$ for $j \in S_{o}$, and $a_{i j} \geq 0$.

Step 2.3: If the inequality (13) holds for each $i \in \mathscr{N}_{j} \cap S_{e}$, where $j \in S_{o}$, then adding them up and noting (11) yields,

$$
u_{j}(m-1) \geq u_{j}(k), \quad k=0, \ldots, m-1, \quad j \in S_{o} .
$$


Hence, for $j \in S_{o}$

$$
u_{j}(m-1) \leq-1
$$

implies

$$
u_{j}(k) \leq-1, \quad k=0, \ldots, m-1 .
$$

A similar argument shows that for $j \in S_{o}$

$$
u_{j}(2 m-1) \geq 1
$$

implies

$$
u_{j}(k) \geq 1, \quad k=m, \ldots, 2 m-1 .
$$

Due to the symmetric property, we can show that if the inequality (13) holds for each $j \in \mathscr{N}_{i} \cap S_{o}$, where $i \in S_{e}$, then adding them up and noting (12) yields, for $i \in S_{e}$,

$$
u_{i}(m-1) \geq 1
$$

implies

$$
u_{i}(k) \geq 1 \quad k=0, \ldots, m-1,
$$

and that

$$
u_{i}(2 m-1) \leq-1
$$

implies

$$
u_{i}(k) \leq-1 \quad k=m, \ldots, 2 m-1 .
$$

To summarize, if we choose

$$
x_{i}(0)=x_{j}(0), \text { for }(i, j) \in \mathscr{E},
$$

where $i \in S_{o}$ and $j \in S_{o}$, or $i \in S_{e}$ and $j \in S_{e}$, then the $2 m$ inequalities for agent $i$, where $i \in \mathscr{V}$ are reduced to only two inequalities, that is, inequalities (14) and (15) for $j \in S_{o}$, and inequalities (16) and (17) for $i \in S_{e}$.

Step 3: It is clear that if there exist $x_{i}(0)$, where $i \in \mathscr{V}$ such that the following two conditions for each $(i, j) \in \mathscr{E}$, where $i \in S_{e}$ and $j \in S_{o}$ are satisfied

$$
\begin{aligned}
& a_{i j}\left\{\alpha\left[x_{i}(m-1)-x_{j}(m-1)\right]+\beta\left[v_{i}(m-1)-v_{j}(m-1)\right]\right\} \\
&= a_{i j}\left\{\alpha \left[x_{i}(0)+(m-1) v_{i}(0)+\frac{(m-1)(m-2)}{2}-x_{j}(0)\right.\right. \\
&\left.-(m-1) v_{j}(0)+\frac{(m-1)(m-2)}{2}\right] \\
&\left.+\beta\left[v_{i}(0)+m-1-v_{j}(0)+m-1\right]\right\} \\
&= a_{i j}\left\{\alpha\left[x_{i}(0)-x_{j}(0)-2 m+2\right]+\beta(m-2)\right\} \leq-1,
\end{aligned}
$$

$a_{i j}\left\{\alpha\left[x_{i}(2 m-1)-x_{j}(2 m-1)\right]+\beta\left[v_{i}(2 m-1)-v_{j}(2 m-1)\right]\right\}$

$$
=a_{i j}\left\{\alpha\left[x_{i}(0)-v_{i}(0)-1-x_{j}(0)+v_{j}(0)-1\right]\right.
$$$$
\left.+\beta\left[v_{i}(0)+1-v_{j}(0)+1\right]\right\}
$$$$
=a_{i j}\left\{\alpha\left[x_{i}(0)-x_{j}(0)+m-2\right]-\beta(m-2)\right\} \geq 1,
$$

then $u_{j}(m-1) \leq-1$ and $u_{j}(2 m-1) \geq 1$ for $j \in S_{o}$, and $u_{i}(m-1) \geq 1$ and $u_{i}(2 m-1) \leq-1$ for $i \in S_{e}$.
These two inequalities are equivalent to

$$
\begin{gathered}
\frac{1}{a_{i j}}+(\beta-\alpha)(m-2) \leq \alpha\left(x_{i}(0)-x_{j}(0)\right) \\
\leq 2 \alpha(m-1)-\beta(m-2)-\frac{1}{a_{i j}}
\end{gathered}
$$

for each $(i, j) \in \mathscr{E}$, where $i \in S_{e}$ and $j \in S_{o}$. We see that suitable $x_{i}(0)$, where $i \in \mathscr{V}$ exist if and only if

$$
\frac{1}{a_{i j}}+(\beta-\alpha)(m-2) \leq 2 \alpha(m-1)-\beta(m-2)-\frac{1}{a_{i j}},
$$

for each $(i, j) \in \mathscr{E}$, where $i \in S_{e}$ and $j \in S_{o}$. This for $m>2$ is equivalent to

$$
\beta \leq \frac{3 m-4}{2 m-4} \alpha-\frac{1}{a_{i j}(m-2)} .
$$

If we take the value of $m$ to be very large, we obtain that

$$
\beta \leq \lim _{m \rightarrow+\infty}\left[\frac{3 m-4}{2 m-4} \alpha-\frac{1}{a_{i j}(m-2)}\right]=\frac{3}{2} \alpha .
$$

Therefore for any $\alpha$ and $\beta$ which satisfy the condition (7), if

$$
m \geq \frac{4(\alpha-\beta)+\frac{2}{\bar{a}}}{3 \alpha-2 \beta}
$$

where

$$
\bar{a}=\min _{\substack{(i, j) \in \mathscr{E} \\ i \in S_{e}, j \in S_{o}}} a_{i j}
$$

then all the inequalities (20) are satisfied.

In the above analysis, we have seen that this implies that the multi-agent system (3) under $T=2 m$, where $m$ satisfies (22), if initial conditions $x_{i}(0)$ satisfy (19), $v_{i}(0)=-\frac{m}{2}$ for $i \in S_{e}$, and $v_{i}(0)=\frac{m}{2}$ for $i \in S_{o}$.

Remark 1 For cyclic topologies, we need equalities (18) on the initial conditions $x_{i}(0)$ for achieving periodic behavior. For tree topologies, $(i, j) \notin \mathscr{E}$, if $i \in S_{o}$ and $j \in S_{o}$, or $i \in S_{e}$ and $j \in S_{e}$, therefore, the last two terms in (10) are vanishing. Hence, for achieving periodic behavior, we have less restrictive conditions on the initial conditions $x_{i}(0)$, in the form of inequalities (19) rather than equalities (18) as in the case of cyclic topologies.

Remark 2 The sufficient condition (7) for achieving periodic behaviors implies that the condition (6) for achieving global consensus has a necessary aspect since the condition (7) is comparable with the condition (6).

\section{An ILlustrative EXAMPle}

In this section, we illustrate the result in Section IV for a network with $N=7$ agents. The network topology is given by the undirected weighted graph depicted in Fig. 1.

Let us choose $\alpha=0.4$ and $\beta=0.42$ such that (7) is satisfied. It is also easy to see that $\bar{a}=a_{36}=0.5$, and therefore we choose $m=11$ such that (22) is satisfied. The 


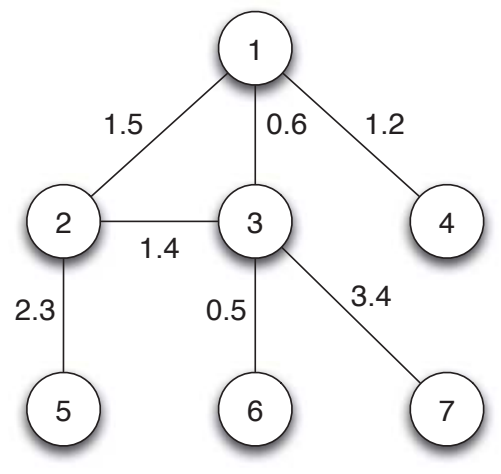

Fig. 1. Network with seven agents

multi-agent system has a periodic solution of period $T=22$ for the following initial conditions:

$$
\left\{\begin{array}{c}
2.1167 \leq x_{1}(0)-x_{2}(0) \leq 8.8833 \\
4.6167 \leq x_{1}(0)-x_{3}(0) \leq 6.3833 \\
2.5333 \leq x_{1}(0)-x_{4}(0) \leq 8.4667 \\
1.5370 \leq x_{5}(0)-x_{2}(0) \leq 9.4630 \\
5.4500 \leq x_{6}(0)-x_{3}(0) \leq 5.5500 \\
1.1852 \leq x_{7}(0)-x_{3}(0) \leq 9.8147 \\
x_{2}(0)=x_{3}(0)
\end{array}\right.
$$

while $v_{i}(0)=-5.5$ for $i \in S_{e}=\{1,5,6,7\}$ and $v_{i}(0)=5.5$ for $i \in S_{o}=\{2,3,4\}$. We then choose

$$
\begin{gathered}
x_{1}(0)=21, x_{2}(0)=16.02, x_{3}(0)=16.02, x_{4}(0)=15, \\
x_{5}(0)=20, x_{6}(0)=21.5, x_{7}(0)=18 .
\end{gathered}
$$

With these initial conditions, The multi-agent system has a periodic solution of period 22, that is, all the agents have periodic solutions of period 22. To make the figure more clear, we have only included the state trajectories for agents 1, 2, 5 and 7 in Fig. 2. The state evolutions for agent 1 are

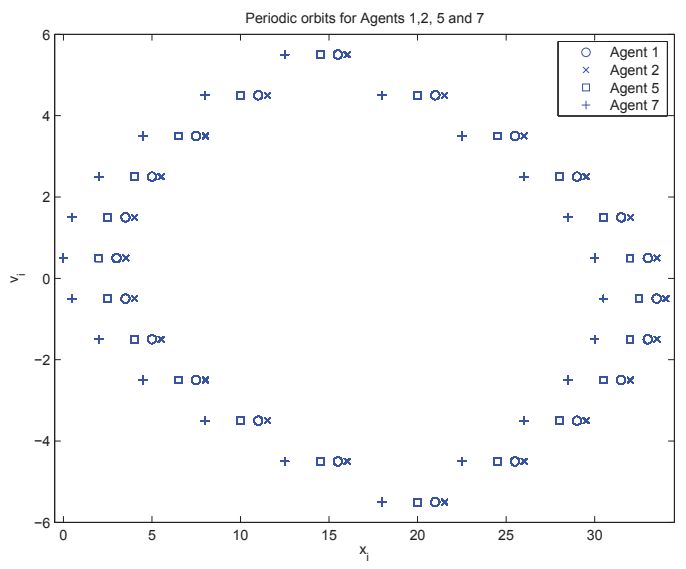

Fig. 2. Periodic behavior of period 22 for the network topology Fig. 1.

also given in Fig. 3 and Fig. 4.

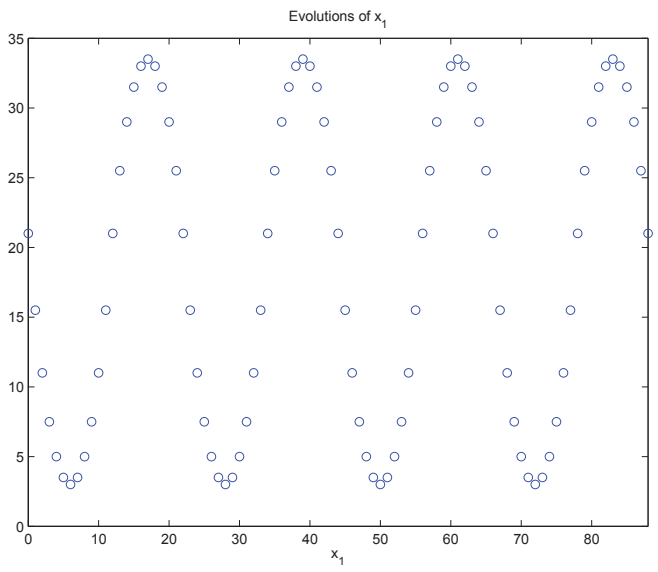

Fig. 3. Evolution of $x_{1}$

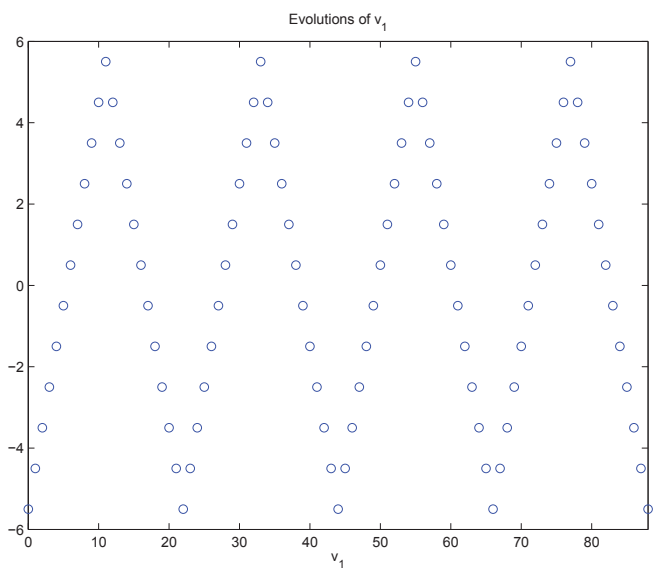

Fig. 4. Evolution of $v_{1}$

\section{CONCLUSION}

In this paper, we show that if the feedback gain parameters of the controllers satisfy a certain condition, then the multiagent system of identical discrete-time double integrators with input saturation constraints under these feedback controllers exhibits periodic behavior. In addition, we identify potential application of the derived framework to robotics' problems such as coverage control and area surveillance.

\section{REFERENCES}

[1] J. Tsitsiklis, "Problems in decentralized decision making and computation," Ph.D. dissertation, MIT, Cambridge, MA, 1984.

[2] N. Lynch, Distributed algorithms. San Mateo, CA: Morgan Kaufmann, 1996.

[3] A. Jadbabaie, J. Lin, and A. S. Morse, "Coordination of groups of mobile autonomous agents using nearest neighbor rules," IEEE Trans. Aut. Contr., vol. 48, no. 6, pp. 988-1001, 2003.

[4] R. Olfati-Saber and R. Murray, "Consensus problems in networks of agents with switching topology and time-delays," IEEE Trans. Aut. Contr., vol. 49, no. 9, pp. 1520-1533, 2004. 
[5] L. Moreau, "Statbility of multiagent systems with time-dependent communication links," IEEE Trans. Aut. Contr., vol. 50, no. 2, pp. 169-182, 2005.

[6] W. Ren and R. Beard, "Consensus seeking in multiagent systems under dynamically changing interaction topologies," IEEE Trans. Aut. Contr. vol. 50, no. 5, pp. 655-661, 2005.

[7] J. Cortés, "Finite-time convergence gradient flows with applications to network consensus," Automatica, vol. 42, no. 11, pp. 1993-2000, 2006.

[8] S. Tuna, "Synchronizing linear systems via partial-state coupling," Automatica, vol. 44, no. 8, pp. 2179-2184, 2008.

[9] J. Seo, H. Shim, and J. Back, "Consensus of high-order linear systems using dynamic output feedback compensator: Low gain approach," Automatica, vol. 45, no. 11, pp. 2659-2664, 2009.

[10] L. Scardovi and R. Sepulchre, "Synchronization in networks of identical linear systems," Automatica, vol. 45, no. 11, pp. 2557-2562, 2009.

[11] W. Yu, G. Chen, and M. Cao, "Some necessary and sufficient conditions for second-order consensus in multi-agent dynamical systems," Automatica, vol. 46, no. 6, pp. 1089-1095, 2010.

[12] Z. Li, Z. Duan, G. Chen, and L. Huang, "Consensus of multiagent systems and synchronization of complex networks: A unified viewpoint," IEEE Trans. Circ. \& Syst.-I Regular papers, vol. 57, no. 1, pp. 213-224, 2010.

[13] T. Yang, S. Roy, Y. Wan, and A. Saberi, "Constructing consensus controllers for networks with identical general linear agents," Int. J. Robust \& Nonlinear Control, vol. 21, no. 11, pp. 1237-1256, 2011.

[14] P. Wieland, R. Sepulchre, and F. Allgöwer, "An internal model principle is necessary and sufficient for linear output synchronization," Automatica, vol. 47, no. 5, pp. 1068-1074, 2011.

[15] K. You and L. Xie, "Network topology and communication data rate for consensusability of discrete-time multi-agent systems," IEEE Trans. Aut. Contr., vol. 56, no. 10, pp. 2262-2275, 2011

[16] H. Grip, T. Yang, A. Saberi, and A. Stoorvogel, "Output synchronization for heterogeneous networks of non-introspective agents," Automatica, vol. 48, no. 10, pp. 2444-2453, 2012.

[17] C. W. Wu, Synchronization in complex networks of nonlinear dynamical systems. Singapore: World Scientic: Singapore, 2007.

[18] W. Ren and Y. Cao, Distributed coordination of multi-agent networks, ser. Communications and Control Engineering Series. London: Springer Verlag, 2011.

[19] H. Bai, M. Arcak, and J. Wen, Cooperative control design: a systematic, passivity-based approach, ser. Communications and Control Engineering. New York: Springer, 2011.

[20] Y. Li, J. Xiang, and W. Wei, "Consensus problems for linea timeinvariant multi-agent systems with saturation constraints," Control Theory Appl., IET, vol. 5, no. 6, pp. 823-829, 2011.

[21] Z. Meng, Z. Zhao, and Z. Lin, "On global leader-following consensus of identical linear dynamic systems subject to actuator saturation," Syst. \& Contr. Letters, vol. 62, no. 2, pp. 132-142, 2013.

[22] T. Yang, A. Stoorvogel, H. Grip, and A. Saberi, "Semi-global regulation of output synchronization for heterogeneous networks of nonintrospective, invertible agents subject to actuator saturation," 2012, to appear in Int. J. Robust \& Nonlinear Control, available at http: //dx.doi.org/10.1002/rnc.2905.

[23] T. Yang, Z. Meng, D. Dimarogonas, and K. Johansson, "Global consensus in homogeneous networks of discrete-time agents subject to actuator saturation," in European Control Conference, Zurich, Switerland, 2013, pp. 244-249.

[24] M. Martelli, Introduction to discrete dynamical systems and chaos, ser. Discrete Mathematics and Optimization. New York: Wiley, 1999.

[25] T. Yang, A. Stoorvogel, and A. Saberi, "Dynamic behavior of the discrete-time double integrator with saturated locally stabilizing linear state feedback laws," 2012, to appear in Int. J. Robust \& Nonlinear Control, available at http://dx.doi.org/10.1002/rnc.2855.

[26] K. Johansson, A. Rantzer, and K. Åström, "Fast switches in relay feedback systems," Automatica, vol. 35, no. 4, pp. 539-552, 1999.

[27] K. Johansson, A. Barabanov, and K. Åström, "Limit cycles with chattering in relay feedback systems," IEEE Trans. Aut. Contr., vol. 47, no. 9, pp. 1414-1423, 2002.

[28] M. Bernardo, K. Johansson, and F. Vasca, "Self-oscillations and sliding in relay feedback systems: symmetry and bifurcations," Int. J. of Bifurcation and Chaos, vol. 11, no. 4, pp. 1121-1140, 2001.

[29] J. Gonçalves, A. Megretski, and M. Dahleh, "Global stability of relay feedback systems," IEEE Trans. Aut. Contr., vol. 46, no. 4, pp. 550$562,2001$.
[30] A. Pogromsky, T. Glad, and H. Nijmeijer, "On diffusion driven oscillations in coupled dynamical systems," Int. J. of Bifurcation and Chaos, vol. 9, no. 4, pp. 629-644, 1999.

[31] S. Strogatz, "From kuramoto to crawford: Exploring the onset of synchronization in populations of coupled oscillators," Physica D, vol. 143, pp. 1-20, 2000.

[32] F. Dörfler and F. Bullo, "Synchronization and transient stability in power networks and non-uniform kuramoto oscillators," SIAM J. Contr. \& Opt., vol. 50, no. 3, pp. 1616-1642, 2012.

[33] J. Cortés, S. Martínez, T. Karataş, and F. Bullo, "Coverage control for mobile sensing networks," IEEE Transactions on Robotics and Automation, vol. 20, no. 2, pp. 243-255, 2004.

[34] S. Smith, J. Tůmová, C. Belta, and D. Rus, "Optimal path planning for surveillance with temporal logic constraints," International Journal of Robotics Research, vol. 30, no. 14, pp. 1695-1708, 2011.

[35] D. Xie and S. Wang, "Consensus of second-order discrete-time multiagent systms with fixed topology," J. Math. Analysis and Appl., vol. 387 , no. 1, pp. 8-16, 2012. 